\title{
Resultados Clínicos e Hemodinâmicos de Longo Prazo após o Transplante de Coração em Pacientes Pré-Tratados com Sildenafil
}

\author{
Long-Term Clinical and Hemodynamic Outcomes after Heart Transplantation in Patients Pre-Treated with Sildenafil
}

Sofia Lázaro Mendes, ${ }^{10}$ Nadia Moreira, ${ }^{1}$ Manuel Batista, ${ }^{1}{ }^{\circledR}$ Ana Rita Ferreira, ${ }^{1}{ }^{\circledR}$ Ana Vera Marinho, ${ }^{1}{ }^{\circledR}$ David Prieto, ${ }^{1}$ Rui Baptista, ${ }^{1}$ Susana Costa, ${ }^{1}$ Fatima Franco, ${ }^{1}$ Mariano Pego, ${ }^{1}$ Manuel de Jesus Antunes ${ }^{1}$

Centro Hospitalar e Universitario de Coimbra EPE, ${ }^{1}$ Coimbra - Portugal

\section{Resumo}

Fundamento: A resistência vascular pulmonar elevada ainda é um grande problema na seleção de candidatos ao transplante cardíaco.

Objetivo: Nosso objetivo foi avaliar o efeito da administração de sildenafila pré-transplante cardíaco em pacientes com hipertensão pulmonar fixa.

Métodos: $O$ estudo retrospectivo, de centro único, incluiu 300 candidatos a transplante cardíaco consecutivos tratados entre 2003 e 2013. Destes, 95 pacientes tinham hipertensão pulmonar fixa e, dentre eles, 30 pacientes foram tratados com sildenafila e acabaram passando pelo transplante, formando o Grupo A. O Grupo B incluiu 205 pacientes sem hipertensão pulmonar que passaram pelo transplante cardíaco. A hemodinâmica pulmonar foi avaliada antes do transplante, 1 semana $\mathbf{1}$ ano após o transplante. A taxa de sobrevivência foi comparada entre os grupos. Neste estudo, um $P$ valor $<0,05$ foi considerado estatisticamente significativo.

Resultados: Após o tratamento com sildenafila, mas antes do TxC, a RVP (-39\%) e a PAPs (-10\%) diminuíram significativamente. A PAPs diminuiu após o TxC em ambos os grupos, mas permaneceu significativamente alta no grupo $A$ em relação ao grupo $B(40,3 \pm 8,0 \mathrm{mmHg}$ versus $36,5 \pm 11,5 \mathrm{mmHg}, \mathrm{P}=0,022)$. Um ano após o TxC, a PAPs era 32,4 $\pm 6,3 \mathrm{mmHg}$ no Grupo A versus $30,5 \pm 8,2 \mathrm{mmHg}$ no Grupo $B(P=0,274)$. $O$ índice de sobrevivência após o TxC 30 dias (97\% no grupo A versus $96 \%$ no grupo B), 6 meses (87\% versus $93 \%$ ) e um ano (80\% versus $91 \%$ ) após o TxC não foi estatisticamente significativo (Log-rank $\mathbf{P}=0,063$ ). Depois do primeiro ano, o índice de mortalidade era similar entre os dois grupos (sobrevivência condicional após 1 ano, Log-rank $p=0,321$ ).

Conclusão: Nos pacientes com HP pré-tratados com sildenafila, a hemodinâmica pós-operatória inicial e o prognóstico são numericamente piores em pacientes sem HP, mas depois de 1 ano, a mortalidade em médio e longo prazo são semelhantes. (Arq Bras Cardiol. 2021; 116(2):219-226)

Palavras-chave: Resistência Vascular; Transplante de Coração; Hipertensão Pulmonar; Citrato de Sildenafila; Inibidores da Fosfodiesterase 5; Disfunção Ventricular Direita.

\footnotetext{
Abstract

Background: Elevated pulmonary vascular resistance remains a major problem for heart transplant (HT) candidate selection.

Objective: This study sought at assess the effect of pre-HT sildenafil administration in patients with fixed pulmonary hypertension.

Methods: This retrospective, single-center study included 300 consecutive, HT candidates treated between 2003 and 2013 , in which 95 patients had fixed $\mathrm{PH}$, and of these, 30 patients were treated with sildenafil and eventually received a transplant, forming Group A. Group B included 205 patients without PH who underwent HT. Pulmonary hemodynamics were evaluated before HT, as well as 1 week after and 1 year after HT. Survival was compared between the groups. In this study, a p value $<0.05$ was considered statistically significant.

Results: After treatment with sildenafil but before HT, PVR (-39\%) and SPAP (-10\%) decreased significantly. sPAP decreased after HT in both groups, but it remained significantly higher in group A vs. group $B(40.3 \pm 8.0 \mathrm{mmHg}$ vs $36.5 \pm 11.5 \mathrm{mmHg}, P=0.022)$. One year after HT, sPAP was $32.4 \pm 6.3 \mathrm{mmHg}$ in group $A$ vs $30.5 \pm 8.2 \mathrm{mmHg}$ in group $B(P=0.274)$. The survival rate after HT at
}

Correspondência: Sofia Lázaro Mendes •

Centro Hospitalar e Universitário de Coimbra - Praceta, R. Prof. Mota Pinto Coimbra 3075, Coimbra - Portugal

E-mail: sofialazaromendes@gmail.com

Artigo recebido em 20/01/2019, revisado em 26/11/2019, aceito em 27/12/2019

DOI: https://doi.org/10.36660/abc.20190047 
30 days (97\% in group A versus 96\% in group B), at 6 months (87\% versus 93\%) and at one year (80\% vs 91\%) were not statistically significant (Log-rank $P=0.063$ ). After this first year, the attrition rate was similar among both groups (conditional survival after 1 year, Log-rank $P=0.321)$.

Conclusion: : In patients with severe PH pre-treated with sildenafil, early post-operative hemodynamics and prognosis are numerically worse than in patients without PH, but after 1 year, the medium to long-term mortality proved to be similar. (Arq Bras Cardiol. 2021; 116(2):219-226)

Keywords: Vascular Resistance; Heart Transplantation; Hypertension Pulmonary; Sildenafil Citrate; Phosphodiesterase 5 Inhibitors; Ventricular Dysfunction, Right.

Full texts in English - http://www.arquivosonline.com.br

\section{Introdução}

O transplante cardíaco (TxC) é o padrão ouro do cuidado da insuficiência cardíaca terminal. ${ }^{1}$ Estudos epidemiológicos mostraram que $60-70 \%$ dos pacientes com insuficiência cardíaca (IC) desenvolvem hipertensão pulmonar (HP). ${ }^{2,3} \mathrm{Em}$ um estudo da Mayo Clinic, ${ }^{4}$ detectou-se uma forte associação graduada entre pressão arterial pulmonar sistólica (PAPs) e mortalidade e, por isso, a presença de HP grave é uma das maiores contraindicações ao $\mathrm{TxC}$, devido à disfunção do coração direito pós-operatória. ${ }^{5}$

Pressões elevadas no lado direito em IC geralmente resultam de pressões de enchimento elevadas no ventrículo esquerdo (VE). Portanto, a pressão arterial pulmonar diastólica (PAPd) está firmemente correlacionada com a pressão capilar pulmonar (PCP). ${ }^{6,7}$ Por outro lado, o componente vasorreativo da HP se desenvolve com a HP duradoura. Ele é caracterizado por vasoespasmo, vasoconstrição e alterações morfológicas dos vasos. ${ }^{8,9}$ Nesse caso, a HP persiste, independentemente da diminuição da PCP após o TxC. Refletindo os componentes "fixos" da HP, a resistência vascular pulmonar (RVP) e o gradiente transpulmonar (GTP) são elevados. ${ }^{6}$

A princípio, a HP é reversível por vasodilatadores sistêmicos, mas, posteriormente ela se torna relativamente estática ou "fixa" ${ }^{6,9,10}$ A RVP elevada aumenta a mortalidade no período inicial pós-TxC e continua sendo um grande problema para a seleção dos candidatos. ${ }^{11,12} \mathrm{~A}$ impossibilidade do coração transplantado de se adaptar a HP significativa préexistente geralmente resulta em insuficiência do ventrículo direito (VD), que representa aproximadamente $50 \%$ de todas as complicações cardíacas e até 19\% de todas as mortes precoces no pós-operatório. ${ }^{12,13}$ Por isso, a avaliação correta que a reatividade do sistema vascular pulmonar exerce para a terapia vasodilatadora tem um papel crucial na seleção do candidato. As diretrizes da American Heart Association definem HP fixa como média da pressão arterial pulmonar $(\mathrm{mPAP}) \geq 25 \mathrm{mmHg}$ e RVP $\geq 2,5$ unidades Wood (UW) e/ou GTP $\geq 12 \mathrm{mmHg}$, mesmo depois da testagem com vasodilatador farmacológico. ${ }^{14}$

A sildenafila é um inibidor de fosfodiestarase tipo 5 (PDE5) seletivo e potente que, especificamente, degrada a guanosina monofosfato cíclico, o segundo mensageiro do óxido nítrico nas células musculares lisas vasculares..$^{8,15}$ A sildenafila tem um perfil favorável sem desnaturação do oxigênio ou alterações significativas da frequência cardíaca ou da pressão sanguínea. ${ }^{16}$ Vários estudos de centro único demonstraram efeito hemodinâmico favorável da administração da sildenafila pré-TxC em candidatos ao TxC com HP. ${ }^{12,17}$ Entretanto, há uma escassez de dados sobre os resultados iniciais e de longo prazo sobre esses pacientes de alto risco.

O objetivo deste estudo é comparar o efeito na hemodinâmica inicial do VD e mortalidade após o TxC da administração da sildenafila pré-TxC em pacientes com HP fixa que se qualificaram para o TxC e em pacientes sem HP. Nossa hipótese é de que os pacientes com HP que foram transplantados com o uso de sildenafila têm prognósticos comparáveis aos dos pacientes sem HP.

\section{Métodos}

\section{População do Estudo}

Este estudo de observação, de centro único e retrospectivo incluiu 300 pacientes consecutivos, candidatos a TxC, observados entre novembro de 2003 e dezembro de 2013. A população incluiu 95 pacientes com hipertensão pulmonar fixa. Dentre eles, 30 pacientes foram tratados com sildenafila e acabaram passando pelo transplante, formando o Grupo A. O Grupo B incluiu 205 pacientes sem HP fixa que passaram pelo TxC.

No grupo A, a sildenafila foi administrada via oral a 20 mg t.i.d., durante um período médio de 65 dias (faixa 4 81) antes do TxC. A sildenafila foi bem tolerada em todos os pacientes envolvidos, sem que eventos adversos sérios tivessem sido observados.

\section{Coleta de Dados}

Dados clínicos, laboratoriais e hemodinâmicos foram extraídos usando um software dedicado. Todos os pacientes passaram por um cateterismo do coração direito (CCD) com um cateter Swan-Ganz, pela veia femoral, antes do começarem a usar a sildenafila. O grupo de pacientes que foram expostos à sildenafila passaram por um segundo CCD para avaliar o efeito hemodinâmico da droga. Depois do TxC, as pressões sistólica do ventrículo direito e diastólica final foram registradas durante a primeira biópsia endomiocárdica, que foi realizada 1 semana após o TxC. Um acompanhamento hemodinâmico tardio foi coletado durante o CCD prédefinido 1 ano após o TxC em ambos os grupos.

O débito cardíaco (DC) foi medido pelo método de Fick, e o índice cardíaco (IC) foi calculado dividindo-se o DC pela área da superfície do corpo. A PCP, a PAPs, a PAPd e a mPAP foram medidas automaticamente. RVP e GTP foram calculados utilizando-se as seguintes fórmulas: 
GTP $(\mathrm{mmHg})=\mathrm{mPAP}-\mathrm{PCP} ; \mathrm{RVP}(\mathrm{UW})=\mathrm{GTP} / \mathrm{DC} .{ }^{18} \mathrm{Um}$ acompanhamento foi realizado por um período médio de 6,9 anos (faixa 4,2 - 6,9 anos) por entrevista pessoal na clínica, análise de registros hospitalares e contato telefônico, e foi realizado para todos os pacientes incluídos. A confidencialidade foi sempre respeitada.

\section{Endpoints}

As medidas de resultados coprimários foram (1) Pressão sistólica de VD e pressão diastólica final (a última usada como substituta da função VD) 7 dias após o TxC e (2) a PAPs e RVP, 1 ano após o TxC. O resultado secundário foi a mortalidade global após o TxC. Os endpoints forma comparados entre grupos pré-definidos.

\section{Análise Estatística}

Variáveis contínuas foram distribuídas normalmente e avaliado usando-se o teste de Shapiro-Wilk, e expressas como média \pm desvio padrão, e as com distribuição não normal foram expressas como média (faixa interquartil). Variáveis dicotômicas foram expressas como frequências (porcentagens). Para comparar dados entre os grupos, utilizamos o teste $\mathrm{T}$ de Student (teste T não pareado) para variáveis contínuas, teste Mann-Whitney para dados não contínuos, e teste qui-quadrado (Fisher, conforme apropriado) para dados dicotômicos. O teste de McNemar foi usado para análise de dados categóricos pareados. As curvas de sobrevivência de Kaplan-Meyer foram construídas e comparadas usando o teste Log-rank. A sobrevivência condicional foi avaliada limitandose o grupo de pacientes analisado aos que sobreviveram pelo menos 1 ano. A análise total foi realizada utilizando-se o software STATA 12.0 (College Station, Texas, EUA). Os gráficos foram construídos com o software GraphPad 5.0 (La Jolla, California, EUA). Neste estudo, um $\mathrm{P}$ valor $<0,05$ foi considerado estatisticamente significativo.

\section{Resultados}

Todos os 235 pacientes passaram por TxC com sucesso. As características da linha de base são apresentadas na Tabela 1. A maioria dos pacientes era do sexo masculino, e a média de idade do grupo A foi de 53,6 \pm 10,9 anos, e do grupo B $52,9 \pm 13,4$ anos $(p=0,545)$. A hemodinâmica pré-TxC é apresentada na Tabela 2 e foi significativamente diferente entre os grupos. Os pacientes do grupo A apresentaram hemodinâmica pulmonar mais grave do que os pacientes do grupo B. Após o tratamento com sildenafila, mas antes do TxC, o RVP (-39\%) e a PAPs (-10\%) diminuíram significativamente (Tabela 3).

\section{Dados Peri-TxC e Resultados Pós-TxC}

As medidas de endpoints coprimários, avaliadas 1 semana após o TxC, são apresentadas na Tabela 3. A evolução da PAPs durante o período de acompanhamento em ambos os grupos é apresentada na Figura 1. A PAPs diminuiu após o TxC em ambos os grupos, mas permaneceu significativamente alta no grupo A em relação ao grupo $\mathrm{B}(40,3 \pm 8,0 \mathrm{mmHg}$ versus 36,5 $\pm 11,5 \mathrm{mmHg}, \mathrm{p}=0,022)$. Não foram encontradas diferenças em relação à pressão diastólica final do VD uma semana após o TxC, usado como substituo da disfunção de VD inicial (Tabela 3). Um ano após o TxC, a PAPs era 32,4 $\pm 6,3 \mathrm{mmHg}$ no Grupo A versus 30,5 $\pm 8,2 \mathrm{mmHg}$ no Grupo B $(P=0,274)$ (Tabela 3). A RVP também foi semelhante entre os dois grupos $(1,8 \pm 0,8 \mathrm{mmHg}$ versus $1,8 \pm 1,0 \cup W, \mathrm{p}=0,789)$.

\section{Análise dos Índices de Sobrevivência}

A mortalidade global pós TxC é apresentada na Figura 2 (Log-rank $\mathrm{p}=0,055)$. O índice de sobrevivência após o TxC do grupo A foi $97 \%$ após 30 dias, 87\% após 6 meses, e $80 \%$ após um ano. No grupo B, a sobrevivência após os mesmos períodos foi de $96 \%$, 93\% e 91\%, respectivamente. A diferença no ponto temporal de um ano não foi estatisticamente significativa (Log-rank $p=0,063$ ). Depois do primeiro ano, o índice de mortalidade era similar entre os dois grupos, conforme mostrado na Figura 3 (sobrevivência condicional após 1 ano, Log-rank $p=0,321$ ).

\section{Discussão}

O tratamento de candidatos a TxC com HP fixa com sildenafila garantiu um período pós-operatório bem-sucedido para a maioria dos pacientes para os quais o $\mathrm{TxC}$ havia sido contraindicado inicialmente. Embora apresentassem hemodinâmica pior logo após o TxC, e mortalidade numericamente mais alta durante o primeiro ano, o prognóstico durante o acompanhamento de médio a longo prazo foi semelhante ao dos pacientes de TxC sem HP.

O limite entre HP fixa e reversível não é claro e não há concordância sobre o tempo necessário para se atingir o nível de irreversibilidade teórica ou sobre os melhores parâmetros para definir esse status. ${ }^{12} \mathrm{Em}$ nosso centro, o CCD é utilizado rotineiramente com um teste vasodilatador pois pose ser útil para estabelecer o risco de morte após o TxC. ${ }^{5}$ Uma das variáveis mais úteis para avaliar o risco é a RVP. ${ }^{13}$ Conforme demonstrado por Taylor et al. ${ }^{19}$ a RVP é um indicador independente de morte precoce após o TxC. Esse grupo relatou que o índice de sobrevivência em pacientes de TxC foi significativamente melhor quando o RVP ficava entre 1 e 3 UW, em comparação com pacientes com RVP entre 3 a 5 UW. Pacientes com RVP > 5 UW apresentaram os piores resultados. Em nosso estudo, utilizamos a sildenafila para diminuir a RVP $(3,3 \pm 2,3 \cup W)$, qualificando, dessa forma, os pacientes para o TxC. Na verdade, entre nossos pacientes que foram tratados com sildenafila, a RVP média era significativamente elevada e excluiria a possibilidade de TxC (5,4 \pm 2,3 UW), se não fosse feita nenhuma intervenção. Se esses pacientes não fossem transplantados, seu prognóstico com tratamento médico teria sido ruim, a menos que um dispositivo de assistência ventricular (LVAD, do inglês left ventricular assist device) fosse implantado.

Curiosamente, dois estudos recentes sugeriram que o suporte do LVAD e a descarga mecânica não pulsante contínua do VE pode reverter uma hipertensão pulmonar que anteriormente não respondia a medicação, e tornar os pacientes aptos ao TxC. ${ }^{20,21}$ É interessante notar que a RVP pré-LVAD nesses estudos $(4,3 \pm 1,7$ UW e 4,8 $\pm 1,8 \cup W)$ foi semelhante à do nosso estudo coorte $(5,4 \pm 2,3 \cup W)$. De 


\section{Artigo Original}

\begin{tabular}{|c|c|c|c|}
\hline Característica ${ }^{a}$ & $\begin{array}{c}\text { Grupo A } \\
(n=30)\end{array}$ & $\begin{array}{l}\text { Grupo B } \\
(n=205)\end{array}$ & Valor de $\mathrm{p}^{\mathrm{b}}$ \\
\hline Média de idade, anos & $53,6 \pm 10,9$ & $52,9 \pm 13,4$ & 0,545 \\
\hline Sexo masculino, \% & 86,7 & 76,2 & 0,247 \\
\hline \multicolumn{4}{|l|}{ Etiologia } \\
\hline Isquêmica, \% & 50,0 & 34,0 & 0,346 \\
\hline Idiopática, \% & 36,7 & 56,3 & \\
\hline Hipertrófica, \% & 3,3 & 4,4 & \\
\hline Restritiva, \% & 10,0 & 2,9 & \\
\hline Congênita, \% & 0,0 & 2,4 & \\
\hline \multicolumn{4}{|l|}{ Classe NYHA } \\
\hline III, \% & 33,3 & 36,4 & 0,968 \\
\hline IV, \% & 66,7 & 63,6 & \\
\hline \multicolumn{4}{|c|}{ Parâmetros laboratoriais } \\
\hline Hemoglobina, g/dl & $12,3 \pm 1,8$ & $12,7 \pm 1,7$ & 0,815 \\
\hline Creatinina, mg/dl & $1,4 \pm 1,0$ & $1,3 \pm 0,5$ & 0,060 \\
\hline BNP, pg/ml & 524 [396 - 912] & 625 [306 - 1039] & 0,906 \\
\hline \multicolumn{4}{|l|}{ Parâmetros cardíacos } \\
\hline FEVE, \% & $19,6 \pm 4,5$ & $21,2 \pm 8,4$ & 0,021 \\
\hline \multicolumn{4}{|l|}{ Regurgitação mitral } \\
\hline Leve, \% & 16,0 & 12,5 & 0,703 \\
\hline Moderada, \% & 32,0 & 34,0 & \\
\hline Moderada a Grave, \% & 24,0 & 14,6 & \\
\hline Grave, \% & 24,0 & 31,2 & \\
\hline \multicolumn{4}{|l|}{ Dispositivos cardíacos } \\
\hline CDI, \% & 40,0 & 21,8 & 0,128 \\
\hline CRT, \% & 10,0 & 22,4 & \\
\hline \multicolumn{4}{|l|}{ Sildenafila, pré-TxC } \\
\hline Duração, dias & $65[4-181]$ & & \\
\hline
\end{tabular}

acordo com Perez-Villa et al. ${ }^{22}$ uma estratégia de redução da RVP elevada usando terapia via oral (sildenafila ou bosentana) em pacientes considerados não aptos ao TxC devido à RVP elevada é viável e pode reduzir o risco de disfunção de VD pósoperatória, como também demonstramos em nosso estudo.

Os inibidores de PDE5 têm despertado o interesse no campo da doença do coração esquerdo. ${ }^{6,12}$ Além da terapia padrão de IC, a intervenção com sildenafila pode melhorar os parâmetros hemodinâmicos pulmonares. ${ }^{6,12}$ Esses efeitos favoráveis surgem de sua inibição seletiva de guanosina monofosfato cíclico (GMPc) nos vasos pulmonares, que promove a vasodilatação e menos remodelagem, além de um efeito semelhante ao da milrinona no VD, devido a um processo de diafonia molecular que pode inibir o PDE3 e aumentar a contratilidade do VD. ${ }^{15,18} \mathrm{Em}$ uma meta-análise recente, ${ }^{2}$ identificou-se que o tratamento com sildenafila reduz a RVP em comparação com o uso de placebo (diferença de média ponderada -1,0 UW, p < 0,01). ${ }^{2}$ Nosso estudo também demonstrou que a administração de sildenafila pré-TxC em candidatos ao TxC com HP teve um efeito hemodinâmico positivo ao reduzir a RVP em aproximadamente 2 UW.

A insuficiência circulatória do lado direito é a morbidade a ela associada ainda são uma fonte importante de morte no perioperatório para pacientes de TxC. Pons et al. ${ }^{12}$ também avaliaram os efeito do uso continuado de sildenafila nos resultados clínicos de TxC (acompanhamento médio, 3,4 $\pm 2,1$ anos). Neste estudo, o índice de sobrevivência após o TxC no grupo de pacientes pré-tratados com sildenafila (incluindo apenas 15 pacientes) foi de $87 \%$ após 30 dias. É importante observar que nenhum outro paciente morreu 
Artigo Original

Tabela 2 - Variáveis hemodinâmicas antes do transplante cardíaco em pacientes com (Grupo A) e sem (Grupo B) hipertensão grave

\begin{tabular}{|c|c|c|c|}
\hline Variável & $\begin{array}{c}\text { Grupo A } \\
\text { Média } \pm \text { DP }\end{array}$ & $\begin{array}{c}\text { Grupo B } \\
\text { Média } \pm \text { DP }\end{array}$ & Valor de $\mathrm{p} a$ \\
\hline RVP, UW & $5,4 \pm 2,3$ & $2,7 \pm 1,8$ & $<0,001$ \\
\hline \multicolumn{4}{|l|}{ PAPs (mmHg) } \\
\hline Sistólica & $58,9 \pm 16,4$ & $44,5 \pm 15,2$ & $<0,001$ \\
\hline Diastólica & $23,1 \pm 8,2$ & $19,4 \pm 8,0$ & 0,025 \\
\hline Média & $36,4 \pm 10,7$ & $29,0 \pm 10,3$ & 0,001 \\
\hline $\mathrm{DC}$, litros/min & $3,7 \pm 1,2$ & $3,6 \pm 1,0$ & 0,645 \\
\hline \multicolumn{4}{|l|}{ PS, mmHg } \\
\hline Sistólica & $75,0 \pm 12,2$ & $74,9 \pm 10,8$ & 0,980 \\
\hline $\mathrm{FC}, \mathrm{ppm}$ & $76 \pm 18$ & $76 \pm 16$ & 0,873 \\
\hline
\end{tabular}

PS: pressão sanguínea; FC: frequência cardíaca; DC: débito cardíaco; PAP: pressão arterial pulmonar; RVP: resistência vascular pulmonar; DP: desvio padrão. aFoi usado o teste $T$ de Student.

Tabela 3 - Variáveis hemodinâmicas antes e depois do Transplante cardíaco em pacientes com (Grupo A) e sem (Grupo B) pré-tratamento com sildenafila

\begin{tabular}{|c|c|c|c|c|c|c|c|c|}
\hline & \multicolumn{2}{|c|}{ Qualificação para CCD } & \multicolumn{2}{|c|}{$\begin{array}{c}\text { CCD 3-meses após } \\
\text { sildenafila }\end{array}$} & \multicolumn{2}{|c|}{ BEM 7 dias após o TxC } & \multicolumn{2}{|c|}{ CCD de 1 ano } \\
\hline & PAPs $(\mathrm{mmHg})$ & RVP (UW) & PAPs $(\mathrm{mmHg})$ & RVP (UW) & $\begin{array}{l}\text { VD sistólica } \\
\text { pressão } \\
\text { (mmHg) }\end{array}$ & $\begin{array}{l}\text { VD diastólica final } \\
\text { pressão (mmHg) }\end{array}$ & PAPs (mmHg) & RVP (UW) \\
\hline Sem sildenafila & $44,5(15,2)$ & $2,7(1,8)$ & -- & -- & $36,5(11,5)$ & $7,0(7,1)$ & $30,48(8,23)$ & $1,8(1,0)$ \\
\hline Sildenafila & $58,9(16,4)$ & $5,4(2,3)$ & $52,8(17,1)^{c}$ & $3,3(2,3)^{d}$ & $40,3(8,0)$ & $7,9(5,8)$ & $32,43(6,39)$ & $1,8(0,8)$ \\
\hline Valor de $p^{a}$ & $<0,001$ & $<0,001$ & -- & -- & $0,022^{b}$ & $0,374^{b}$ & 0,274 & 0,789 \\
\hline
\end{tabular}

BEM: biópsia endomiocárdica; RVP: resistência vascular pulmonar; CCD: cateterismo do coração direito; UW: unidades Wood. a Teste $t$ de Student comparando pacientes sem sildenafila pacientes a pacientes tratados com sildenafila. ${ }^{6}$ Teste de McNemmar. ${ }^{\circ} p=0,845$ versus pacientes sem sildenafila. ${ }^{d} p=0,806$ versus pacientes sem sildenafila.

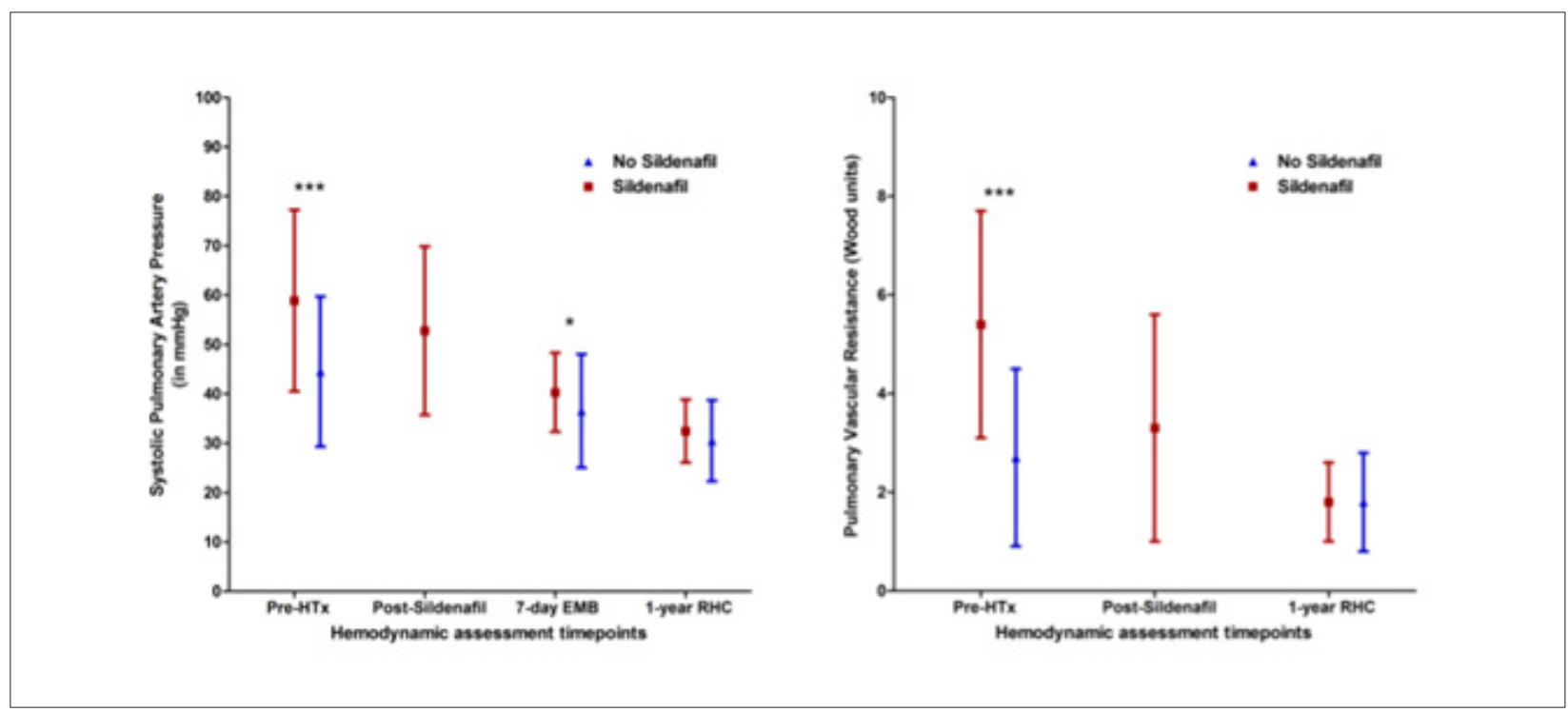

Figura 1 - (Painel esquerdo) Pressão arterial pulmonar sistólica (PAPs, em $\mathrm{mmHg}$ ) em quatro momentos diferentes: linha de base antes do transplante cardíaco (TxC) sem tratamento com sildenafila, antes do TxC com tratamento com sildenafila, logo após o TxC (7 dias) e muito tempo após o TxC (um ano). ${ }^{* *} p<0,001,{ }^{*} p=$ 0,022. (Painel direito) Resistência vascular pulmonar (RVP, em unidades Wood) em três pontos no tempo diferentes: linha de base antes do (TxC) sem tratamento com sildenafila, antes do TxC com tratamento com sildenafila, e mais tarde após o TxC (um ano). ${ }^{* *} p<0,001,{ }^{*} p=0,789$. BEM: biópsia endomiocárdica; TxC: transplante cardíaco; CCD: cateterismo do coração direito. 


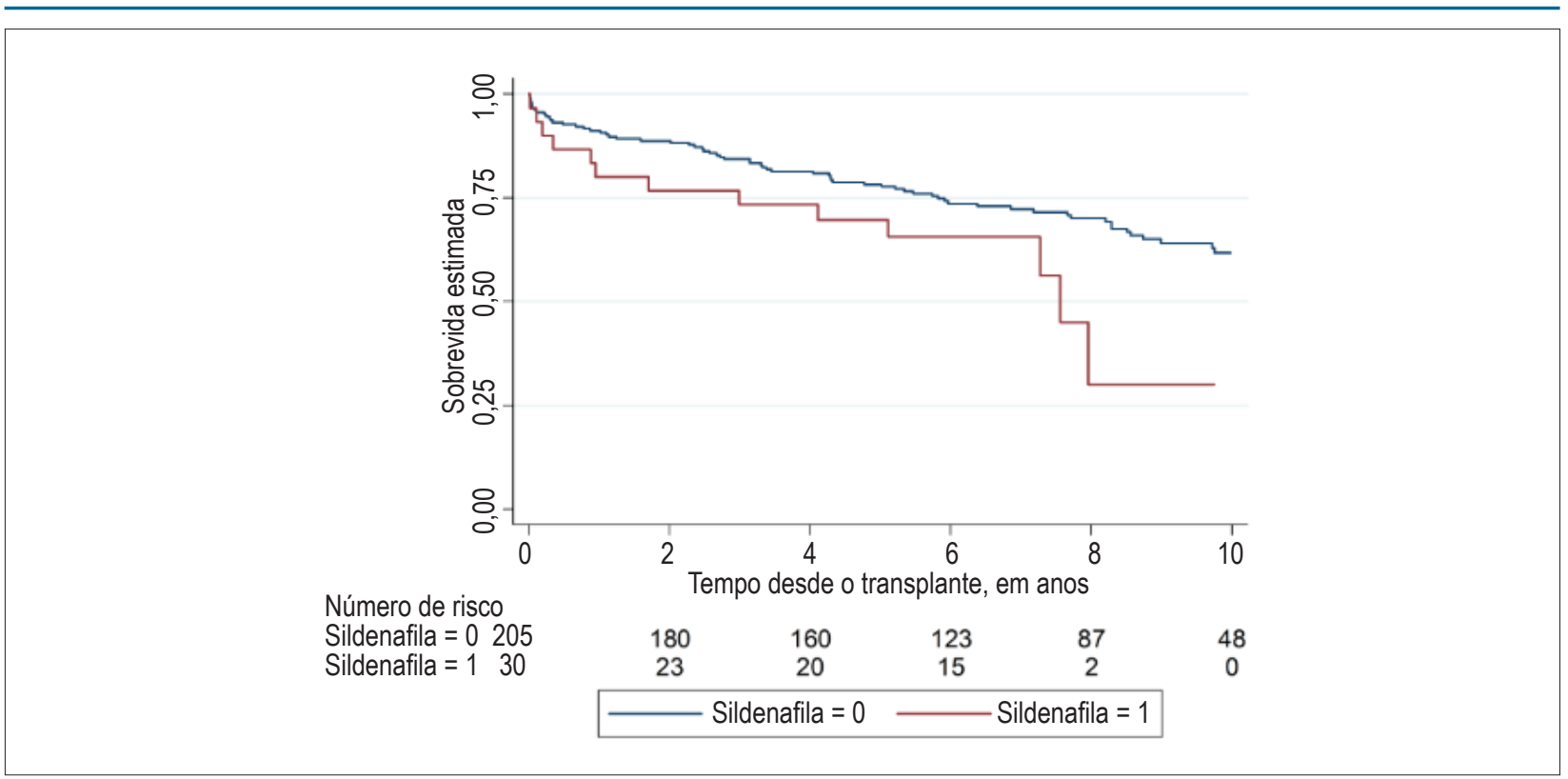

Figura 2 - Análise de Kaplan-Meier da mortalidade global após o transplante de acordo com o grupo de tratamento com sildenafila. Log-rankp =0,063.

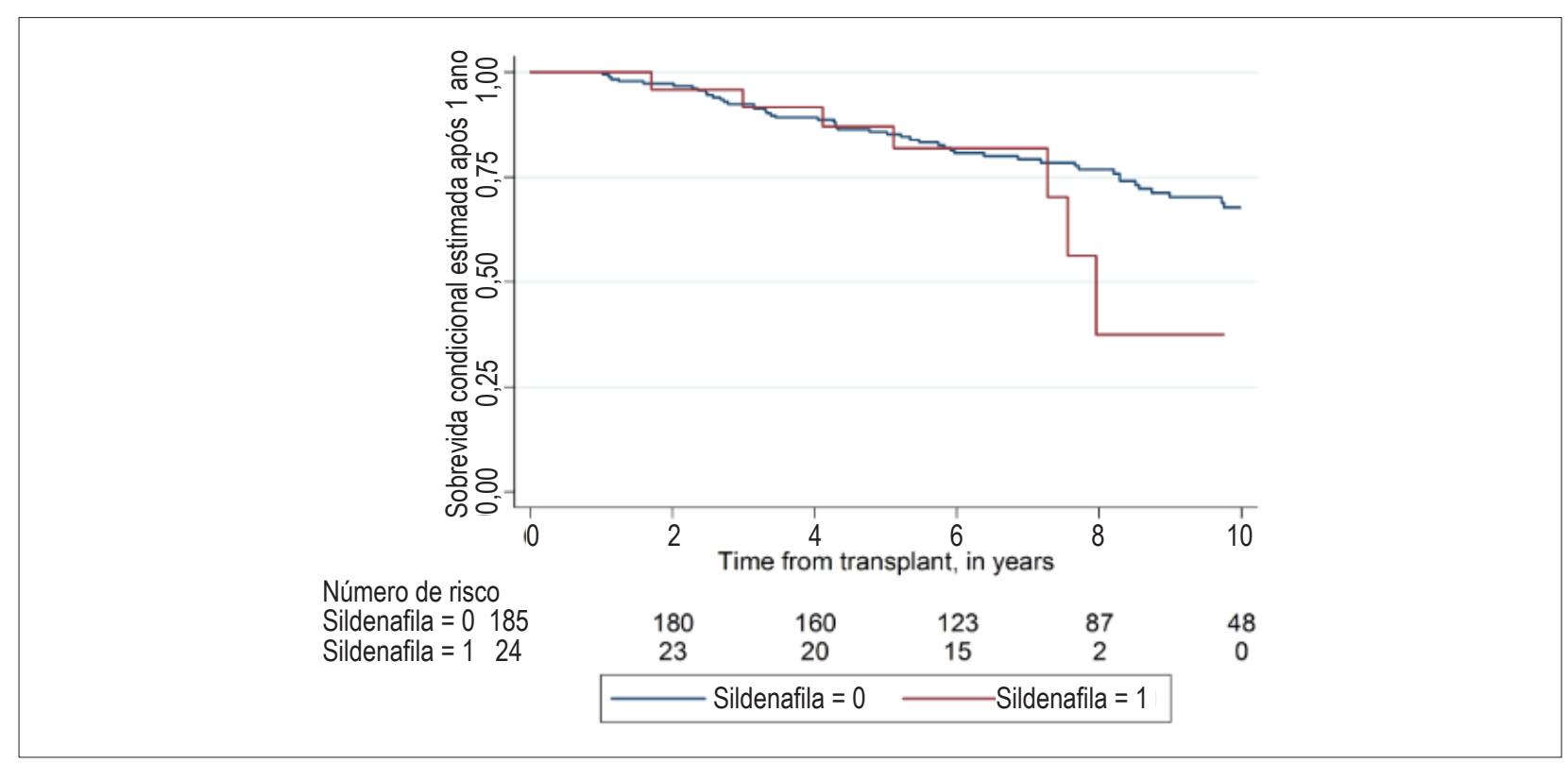

Figura 3 - Análise de Kaplan-Meier, análise de sobrevivência condicional após 1 ano. Log-rank p = 0,321.

durante o período de acompanhamento de 5 anos após o TxC. Comparativamente, o índice de sobrevivência do grupo A foi $97 \%$ após 30 dias e $70 \%$ após cinco anos. Em conformidade com isso, no ISHLT - International Registry for Heart Transplantation, o índice de sobrevivência após 5 anos era de $72 \%$, semelhante a nosso grupo de pacientes com HP fixa pré-tratada com sildenafila. ${ }^{23}$

Por todos esses motivos, uma estratégia usando a sildenafila para reduzir a RVP pode ser considerada uma "terapia de resgate" valiosa em um grupo de pacientes com IC terminal, que não estariam aptos ao TxC de outra forma. Nossos dados mostram que ela está associada a índices de mortalidade no perioperatório e em longo prazo semelhantes aos observados em pacientes sem HP.

\section{Limitações}

As limitações deste estudo incluem sua natureza retrospectiva e não controlada, o que pode condicionar uma seleção tendenciosa. Entretanto, incluímos todos os pacientes que foram transplantados consecutivamente em nosso 
centro, e nenhum paciente foi perdido durante o período de acompanhamento. Além disso, o tamanho de nossa amostra é relativamente pequeno, o que limita o poder estatístico. No entanto, relatamos o que acreditamos ser a maior série existentes de pacientes de TxC pré-tratados com sildenafila. Outra limitação é a ausência de medições diretas da função do VD imediatamente após o TxC. Tentamos compensar esse fato utilizando uma medição hemodinâmica da função do VD coletada 7 dias após o procedimento. Apesar de todas essas limitações, acreditamos que os resultados podem ter validade externa para outras populações com IC avançada, já que os dados demográficos, clínicos e hemodinâmicos estão alinhados com os relatados em outros estudos.

\section{Conclusão}

O uso de sildenafila em candidatos a TxC com HP fixa melhorou a hemodinâmica pulmonar, levando-a a um limiar em que o transplante seria possível. Nesse grupo de pacientes de alto risco, a hemodinâmica pós-operatória inicial e os resultados foram ligeiramente comprometidos, em comparação com pacientes sem HP. Entretanto, após 1 ano, os resultados de médio a longo prazo foram semelhantes entre os grupos. Nossos achados corroboram o conceito de que a sildenafila pode resgatar pacientes previamente inaptos para o TxC

\section{Referências}

1. Ferreira AR, Mendes S, Leite L, Monteiro S, Pego M. Pulse pressure can predict mortality in advanced heart failure. Rev Port Cardiol. 2016;35(4):225-8

2. Wu X, Yang T, Zhou Q, Li S, Huang L. Additional use of a phosphodiesterase 5 inhibitor in patients with pulmonary hypertension secondary to chronic systolic heart failure: a meta-analysis. Eur J Heart Fail. 2014;16(4):444-53.

3. Lam CSP, Roger VL, Rodeheffer RJ, Borlaug BA, Enders FT, Redfield MM Pulmonary hypertension in heart failure with preserved ejection fraction: a community-based study. J Am Coll Cardiol. 2009;53(13):1119-26.

4. Bursi F, McNallan SM, Redfield MM, Nkomo VT, Lam CSP, Weston SA, et al. Pulmonary pressures and death in heart failure: a community study. J Am Coll Cardiol. 2012;59(3):222-31

5. Reichenbach A, Al-Hiti H, Malek I, Pirk J, Goncalvesova E, Kautzner J, et al. The effects of phosphodiesterase 5 inhibition on hemodynamics, functional status and survival in advanced heart failure and pulmonary hypertension: a case-control study. Int J Cardiol. 2013;168(1):60-5.

6. Guglin M, Rajagopalan N, Anaya P, Charnigo R. Sildenafil in heart failure with reactive pulmonary hypertension (Sildenafil HF) clinical trial (rationale and design). Pulm Circ. 2016;6(2):161-7.

7. Moreira N, Baptista R, Costa S, Franco F, Pêgo M, Antunes M. Lowering pulmonary wedge pressure after heart transplant: pulmonary compliance and resistance effect. Arq Bras Cardiol. 2015;105(3):292-300.

8. Guglin M, Khan H. Pulmonary hypertension in heart failure. J Card Fail. 2010;16(6):461-74

9. Guazzi M. Pulmonary hypertension in heart failure preserved ejection fraction: prevalence, pathophysiology, and clinical perspectives. Circ Heart Fail. $2014 ; 7(2): 367-77$.

\section{Contribuição dos Autores}

Concepção e desenho da pesquisa: Mendes SL, Moreira N; Obtenção de dados: Mendes SL, Moreira N, Batista M, Ferreira AR, Marinho AV, Prieto D; Análise e interpretação dos dados: Mendes SL, Moreira N, Batista M, Ferreira AR, Marinho AV, Prieto D, Baptista R, Costa S; Análise estatística: Mendes SL, Baptista R; Redação do manuscrito: Mendes SL, Ferreira AR, Baptista R; Revisão crítica do manuscrito quanto ao conteúdo intelectual importante: Costa S, Franco F, Pego M, Antunes MJ.

\section{Potencial Conflito de Interesses}

Declaro não haver conflito de interesses pertinentes.

\section{Fontes de Financiamento}

O presente estudo não teve fontes de financiamento externas.

\section{Vinculação Acadêmica}

Não há vinculação deste estudo a programas de pós-graduação.

\section{Aprovação Ética e Consentimento Informado}

Este artigo não contém estudos com humanos ou animais realizados por nenhum dos autores.

10. Hefke T, Zittermann A, Fuchs U, Schulte-Eistrup S, Gummert JF, Schulz U Bosentan effects on hemodynamics and clinical outcome in heart failure patients with pulmonary hypertension awaiting cardiac transplantation. Thorac Cardiovasc Surg. 2012;60(1):26-34.

11. Costard-Jäckle A, Fowler MB. Influence of preoperative pulmonary artery pressure on mortality after heart transplantation: testing of potential reversibility of pulmonary hypertension with nitroprusside is useful in defining a high risk group. J Am Coll Cardiol. 1992;19(1):48-54.

12. Pons J, Leblanc MH, Bernier M, Cantin B, Bourgault C, Bergeron S, et al. Effects of chronic sildenafil use on pulmonary hemodynamics and clinical outcomes in heart transplantation. J Heart Lung Transplant. 2012;31(12):1281-7

13. Taylor DO, Stehlik J, Edwards LB, Aurora P, Christie JD, Dobbels F, et al. Registry of the International Society for Heart and lung transplantation: Twenty-sixth Official Adult Heart Transplant Report-2009. J Heart Lung Transplant. 2009;28(10):1007-22.

14. Costanzo MR, Augustine S, Bourge R, Bristow M, O'Connell JB, Driscoll $D$, et al. Selection and treatment of candidates for heart transplantation. A statement for health professionals from the Committee on Heart Failure and Cardiac Transplantation of the Council on Clinical Cardiology, American Heart Association. Circulation. 1995;92(12):3593-612.

15. Schwartz BG, Levine LA, Comstock G, Stecher VJ, Kloner RA. Cardiac uses of phosphodiesterase-5 inhibitors. J Am Coll Cardiol. 2012;59(1):9-15.

16. Lewis GD, Lachmann J, Camuso J, Lepore JJ, Shin J, Martinovic ME, et al. Sildenafil improves exercise hemodynamics and oxygen uptake in patients with systolic heart failure. Circulation. 2007;115(1):59-66.

17. Groote P, El Asri C, Fertin M, Goéminne C, Vincentelli A, Robin E, et al Sildenafil in heart transplant candidates with pulmonary hypertension. Arch Cardiovasc Dis. 2015;108(6-7):375-84 


\section{Artigo Original}

18. Galie N, HumbertM, VachierycJL, Gibbs S, Lang I, Torbicki A, etal. 2015 ESC/ ERS Guidelines for the diagnosis and treatment of pulmonary hypertension: The Joint Task Force for the Diagnosis and Treatment of Pulmonary Hypertension of the European Society of Cardiology (ESC) and the European Respiratory Society (ERS): Endorsed by: Association for European Paediatric and Congenital Cardiology (AEPC), International Society for Heart and Lung Transplantation (ISHLT). Eur Heart J. 2015;37(1):67-119.

19. Trulock EP, Edwards LB, Taylor DO, Boucek MM, Keck BM, Hertz MI. The Registry of the International Society for Heart and Lung Transplantation: twenty-first official adult lung and heart-lung transplant report--2004. J Heart Lung Transplant. 2004;23(7):804-15.

20. Alba AC, Rao V, Ross HJ, Jensen AS, Sander K, Gustafsson F, et al. Impact of fixed pulmonary hypertension on post-heart transplant outcomes in bridgeto-transplant patients. J Heart Lung Transplant. 2010;29(11):1253-8.
21. Etz CD, Welp HA, Tjan TDT, Hoffmeier A, Weigang E, Scheld HH, et al. Medically refractory pulmonary hypertension: treatment with nonpulsatile left ventricular assist devices. Ann Thorac Surg. 2007;83(5):1697-705.

22. Perez-Villa F, Farrero M, Sionis A, Castel A, Roig E. Therapy with sildenafil or bosentan decreases pulmonary vascular resistance in patients ineligible for heart transplantation because of severe pulmonary hypertension. J Heart Lung Transplant. 2010;29(7):817-8.

23. Yusen RD, Edwards LB, Dipchand AI, Goldfarb SB, Kucheryavaya AY, Levvey BJ, et al. The Registry of the International Society for Heart and Lung Transplantation: Thirty-third Adult Lung and Heart-Lung Transplant Report-2016; Focus Theme: Primary Diagnostic Indications for Transplant. J Heart Lung Transplant. 2016;35(10):1170-84. 